\title{
Lobar pneumonia in Northern Zambia: clinical study of 502 adult patients
}

\author{
SC ALLEN
}

From the Department of Medicine, Kasama General Hospital, Zambia

\begin{abstract}
From July 1981 to January 1983502 adults were treated for lobar or segmental pneumonia in a general hospital in Northern Zambia. Consolidation was present in either the right or the left lower lobe in $78.8 \%$, the right middle lobe in $10.2 \%$ and either the right or the left upper lobe in $10.7 \%$. Penicillin treatment failed to give an improvement in $10.7 \%$, many of whom recovered after receiving either gentamicin or kanamycin. A high risk of death was associated with an age of 65 years or over, absence of pyrexia, absence of a leucocyte response, disease affecting multiple lobes, irreversible hypotension, underlying neoplasm, and failure to respond to penicillin treatment ( $p<0.001$ in all cases). Penicillin remains the initial treatment of choice for lobar pneumonia in rural central Africa. Mortality can probably be reduced by inpatient treatment of as many patients with pneumonia as local circumstances allow, and by adding broad spectrum treatment in patients who show no improvement after 48 hours of penicillin treatment.
\end{abstract}

Pneumonia is a major cause of morbidity and mortality in Central Africa, and during the period of this study it was second only to malaria as a reason for admission to an adult medical bed at Kasama General Hospital in the Northern Province of Zambia. Few studies of the clinical pattern of pneumonia in this region, however, have been published. In West Africa clinical and laboratory studies ${ }^{1-4}$ have shown Streptococcus pneumoniae to be the most common aetiological agent. Mycoplasma pneumoniae was detected in $17 \%$ of a series of cases of pneumonia in Nigeria, ${ }^{1}$ though it was emphasised that laboratory methods which are likely to be available in developing countries would not be able to distinguish mycoplasmal pneumonia from other pneumonias in time to influence initial treatment. About $10 \%$ of patients with pneumonia in the tropics do not respond to treatment with penicillin ${ }^{5-6}$ and in one study ${ }^{4} 31 \%$ did not respond. It is clear that further studies could help to shape treatment guidelines which would be of value to health care workers dealing with pneumonia in the remote districts of Central Africa and similar environments. This study was

Address for reprint requests: Teaching Unit 4, Withington Hospital, West Didsbury, Manchester.

Accepted 26 March 1984 undertaken to determine the features of pneumonia in the region, with particular emphasis on the response to penicillin treatment and factors associated with a high risk of death.

\section{Patients and methods}

From July 1981 to January 1983724 adult patients suffering from lower respiratory tract infection were treated at Kasama General Hospital. Two hundred and twenty two were excluded from the study because of an initial diagnosis other than lobar or segmental pneumonia; most of these had pulmonary tuberculosis, lung abscess, empyema thoracis, or bronchitis. Patients who refused inpatient treatment were excluded. Twenty seven of the patients included in the study admitted to having taken an antibiotic immediately before admission; 15 patients had taken tetracycline, two ampicillin and one sulphadoxine, and in nine patients the type of antibiotic taken was uncertain.

The remaining 502 adults ( 362 men, 140 women; 501 black, one white) were included in the study as they had a diagnosis of lobar or segmental pneumonia. The age range was 10-89 (mean 39) years; some ages were estimated. Most were subsistence farmers who lived in thatched, mud walled houses grouped into small villages. 
After admission to hospital a history was obtained and a physical examination performed; the findings were reviewed by the author within 24 hours. Rectal temperature on admission was measured before penicillin was given and was repeated immediately if the first measurement was $37 \cdot 0^{\circ} \mathrm{C}$ or less.

A chest radiograph was taken shortly after admission in 459 cases. A further 43 patients who had a clearcut history and signs of lobar consolidation, and who responded promptly to penicillin treatment, were included in the study despite not having had a chest radiograph.

Haemoglobin and total blood leucocyte measurements were performed on 477 patients, and blood films were examined for the presence of malaria parasites.

A pair of blood samples was taken for standard bacterial culture before antibiotic treatment from only 132 patients. These were generally the more seriously ill patients. Two hundred and forty seven patients were able to produce a sputum sample suitable for Gram stain examination and most of these samples were also examined by Ziehl-Neelsen staining for evidence of tuberculosis.

All patients were initially treated with intramuscular benzylpenicillin $0 \cdot 5-1 \cdot 0$ million units six hourly or with procaine penicillin 0.6 million units daily. Supportive treatment, including oxygen and blood transfusion, and treatment for coexisting conditions were given as necessary, though no patients received intermittent positive pressure ventilation.

Patients who did not show clinical evidence of improvement within about 48 hours of starting penicillin treatment were given an additional parenteral antibiotic, either gentamicin or kanamycin (according to availability) after exclusion and, when necessary, treatment of concomitant malaria. Those who had sputum, blood culture, or strong radiological evidence suggestive of staphylococcal pneumonia were given intramuscular cloxacillin. Eight patients who remained ill and febrile when receiving penicillin and an aminoglycoside were treated by the addition of tetracycline after exclusion of malaria.

In patients who responded promptly to penicillin the treatment was changed to oral phenoxymethylpenicillin $250 \mathrm{mg}$ six hourly when body temperature returned to normal. Most patients were discharged from hospital then and given oral phenoxymethylpenicillin for at least five days. The mean duration of inpatient treatment was 3.4 days in the penicillin responsive patients and 8.7 days in those requiring additional antibiotics. The more severely ill patients and those given additional antibiotics remained on parenteral treatment as inpatients. Patients who proved to be hypersensitive to penicillin were treated with oral erythromycin (six patients).

All patients were offered a follow up outpatient appointment, although fewer than a quarter attended. Most of these patients lived close to the hospital.

Statistical analysis was carried out with the $\chi^{2}$ test (with Yates's correction for small numbers where appropriate) or in some cases Fisher's exact probability test.

\section{Results}

The main presenting symptoms and physical signs are summarised in table 1 . The lack of typical signs in a small proportion (6\%) of patients emphasises the value of chest radiography in this context. Only 16 patients $(3.2 \%)$ were emaciated, and in most of these underlying malignancy or tuberculosis was diagnosed subsequently.

Three hundred and ninety six patients $(78.8 \%)$ had either right or left lower lobe pneumonia and 51 $(10.2 \%)$ had consolidation in the right middle lobe, and in $54(10.7 \%)$ either the right or the left upper lobe was affected. Twelve $(2.4 \%)$ had multiple lobes affected and one had lingular consolidation.

One hundred (19.9\%) had a haemoglobin concentration of less than $10 \mathrm{~g} / \mathrm{dl} ; 68(68 \%)$ of these were women, most of whom were either pregnant or breast feeding, and many also had intestinal hookworm infestation. Though the body weight of most of the anaemic patients was not low, and most had a reasonable quantity of subcutaneous fat, the high incidence of anaemia in the study group probably reflects widespread suboptimal nutrition in the population being studied.

Blood leucocyte measurements are summarised in figure 1 . There was a significantly higher mortality rate among patients who had peripheral blood leucocyte counts of less than $4.0 \times 10^{9} / 1$ at the time of admission (10/27 died) than among those with leucocyte counts of $4.0 \times 10^{9} / 1$ or more $(11 / 450$ died) $(\mathrm{p}<0.001)$.

Body temperatures at the time of admission are summarised in figure 2 . A relatively high mortality rate was observed in patients who had a body temp-

Table 1 Main presenting symptoms and signs in 502 patients with lobar or segmental pneumonia

\begin{tabular}{ll}
\hline Symptoms or sign & No (\%) \\
\hline Cough & $466(93)$ \\
Pleuritic pain & $313(62)$ \\
Rigors & $229(46)$ \\
Headache & $206(41)$ \\
Crepitations over the affected lobe & $417(83)$ \\
Bronchial breath sounds & $264(53)$ \\
Aegophony & $225(45)$ \\
\hline
\end{tabular}




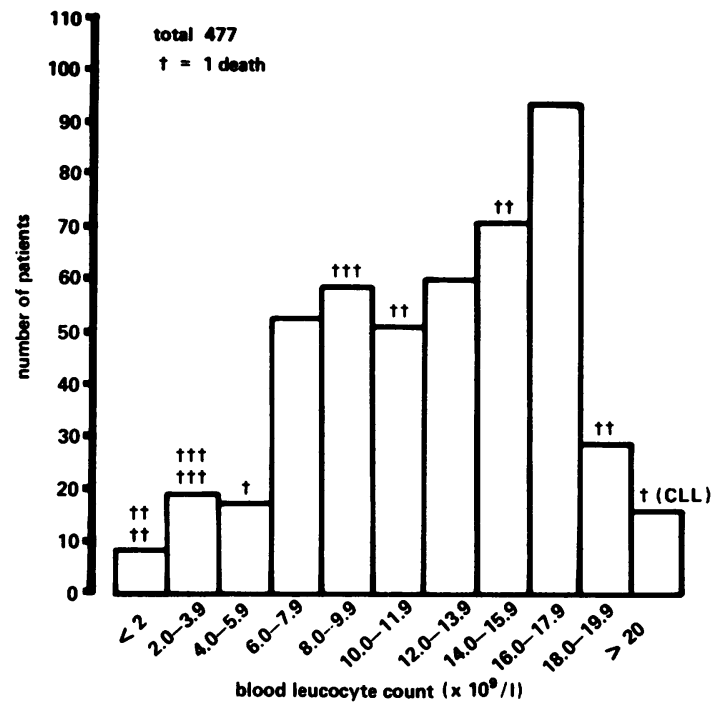

Fig 1 Blood leucocyte count at the time of admission in patients with lobar or segmental pneumonia (total 477). CLL_chronic lymphatic leukaemia.

erature of less than $37^{\circ} \mathrm{C}(9 / 42$ died compared with $12 / 460$ with a temperature of $37^{\circ} \mathrm{C}$ or more, $\mathrm{p}<$ $0 \cdot 001$ ). All four patients in whom body temperature failed to rise to normal with treatment died. Four afebrile patients had taken an antibiotic before admission; all survived. There was no significant increase in mortality rate among patients with body temperatures greater than $40^{\circ} \mathrm{C}(5 / 167$ died compared with $16 / 335$ with temperatures of $39.9^{\circ} \mathrm{C}$ or less.

Table 2 shows the results of blood culture and sputum Gram and Ziehl-Neelsen staining in relation to the response to penicillin treatment. It is noteworthy that 33 patients with underlying pulmonary tuberculosis presented with superimposed pneumonia.

Thirty three patients $(7.4 \%)$ developed serious complications but survived after receiving appropriate treatment. Twelve patients had sickle cell crises;

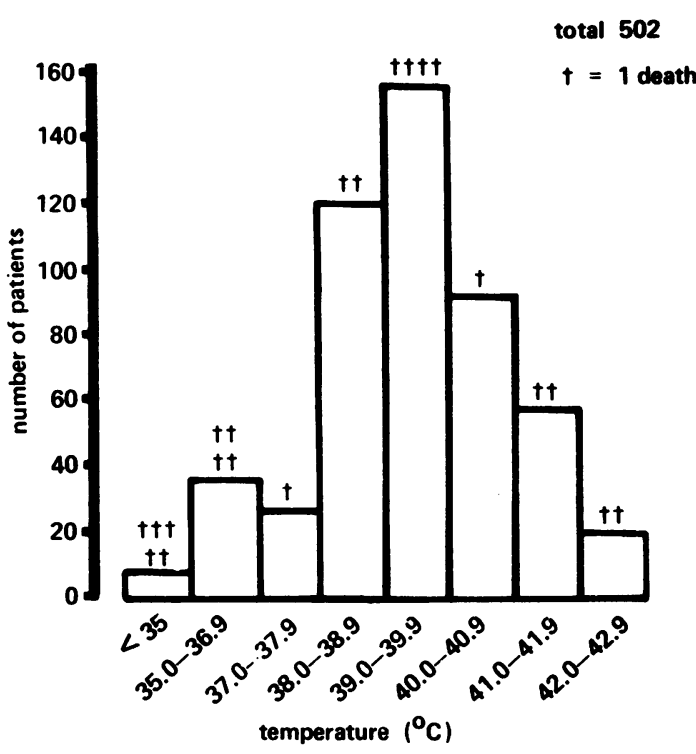

Fig 2 Body temperature at the time of admission in patients with lobar or segmental pneumonia (total 502).

six had hypotensive episodes. There were two to four cases each of pyogenic arthritis, meningitis, atrial fibrillation, and empyema thoracis and single cases of pneumothorax, cavernous sinus thrombosis, bacterial endocarditis, and haemolysis of uncertain cause. One patient with jaundice died at a later date from primary hepatoma. Eighteen $(54.5 \%)$ patients in this group did not respond promptly to penicillin and consequently received either gentamicin or kanamycin.

Twenty one patients $(4.2 \%)$ died shortly after admission from causes which appeared to be directly related to the pneumonia. Eight of these had multiple lobes affected, and it was considered that a long delay in presenting to the hospital was a contributing factor in seven. Nine had systolic blood pressures of less than $90 \mathrm{~mm} \mathrm{Hg}$, which did not rise in response to intravenous fluids, hydrocortisone, and oxygen. Two had associated meningitis and three were bac-

Table 2 Relation between sputum Gram staining and Ziehl-Neelsen staining, blood culture results, and response to penicillin

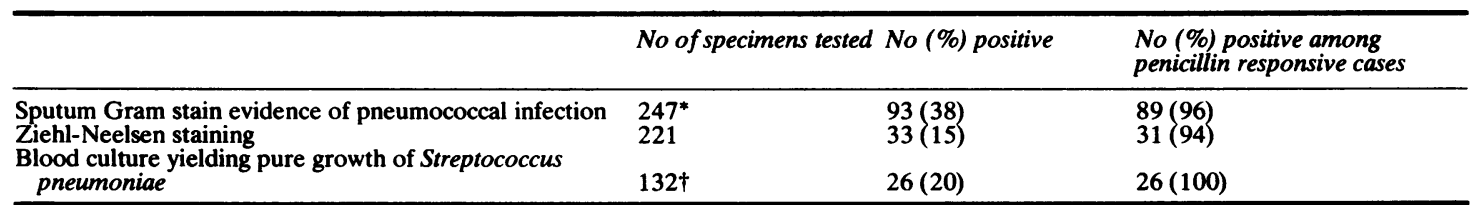

*In addition, organisms resembling Staphylococcus aureus were seen in three sputa and Gram negative bacilli in eight sputa.

†A pure growth of Staph aureus was obtained in one patient, in whom the sputum Gram stain result was suggestive of Staph aureus. 
teraemic (seven tested), though a pure growth of Streptococcus pneumoniae was not isolated in these cases.

A further seven patients (1.4\%) died later in hospital after responding satisfactorily to treatment for pneumonia; of these, two had bronchogenic carcinoma, three hepatoma and one gastric carcinoma, and one patient suffered a stroke.

It was not possible to determine the number of deaths, if any, which occurred in the patients who were discharged after responding to treatment. This was due mainly to low attendance at follow up.

Table 3 summarises the factors which were associated with a significantly higher risk of death. Patients with combinations of risk factors had a very high mortality rate, though the numbers were too few to allow statistical analysis.

Four hundred and forty patients (87.6\%) had a satisfactory response to treatment with penicillin and went on to recover without an additional antibiotic being given. Fifty four $(10.7 \%)$ did not show signs of improvement within 48 hours of starting penicillin treatment and were given an additional antibiotic. A fall in body temperature and an improvement in the feeling of wellbeing with a return of appetite were the most usually accepted indications of a therapeutic response to penicillin treatment, though in many patients in this category the body temperature did not return completely to normal for up to four days.

\section{Discussion}

The study clearly shows that lobar or segmental pneumonia is a major cause of ill health in rural central Africa and that most such infections respond promptly to penicillin treatment. Nevertheless, $\mathbf{1 0 . 7 \%}$ of pneumonias were found to be unresponsive to penicillin, this proportion being similar to that found in other parts of the developing world. ${ }^{56}$ The aetiological agent was demonstrated in only a small proportion of patients, though it is likely, to judge by the overall clinical picture, that most had pneumococcal infection. The types of infection in the penicillin unresponsive pneumonias are not known, and a bacteriological and serological study of such patients would provide useful further information, particularly since the mortality rate was higher in that group.

Sofowara and Onadeko found a $20 \%$ death rate among Nigerians with pneumonia. ${ }^{3}$ The overall mortality in the present study was low by comparison. The likely reasons for this are fourfold. Firstly, the present study included a large proportion of patients with minor degrees of lung consolidation, in whom the risk of death was small, as it was my policy to treat all such patients as inpatients, whereas in many parts of the developing world patients with early or minimal pneumonia are treated as outpatients and are presumably not included in hospital based studies. Secondly, this study did not include young children, in whom the mortality from pneumonia, particularly during measles and whooping cough epidemics, is high in this region. Thirdly, patients with other forms of severe lower respiratory tract infection, such as lung abscess and extensive pulmonary tuberculosis, were excluded from the study (the mortality rate in such patients was about $30 \%$ in this hospital). A fourth possible reason is related to local custom: it is often considered more appropriate for an elderly person who appears to be terminally ill to remain in his or her village to die; thus the proportion of elderly patients (in whom the risk of death from pneumonia is high) included in the study may have been reduced.

A large number of patients were found to have underlying pulmonary tuberculosis, though in most cases the pneumonia responded to penicillin and was therefore presumably due to superimposed acute bacterial infection. It is probably worthwhile, whenever possible, examining sputum from patients with pneumonia in this region to look for tuberculosis, although Gram staining the sputum seems to

Table 3 Factors associated with an increased risk of death in patients with lobar or segmental pneumonia

\begin{tabular}{|c|c|c|c|c|}
\hline \multirow[t]{2}{*}{ Feature } & \multicolumn{2}{|c|}{ Patients with the feature } & \multicolumn{2}{|c|}{ Patients without the feature } \\
\hline & No of patients & No $(\%)$ of deaths & No of patients & No (\%) of deaths \\
\hline $\begin{array}{l}\text { Age }>65 \text { y } \\
\text { Temperature }<37^{\circ} \mathrm{C} \\
\text { Leucocyte count }<4.0 \times 10^{9} / 1 \text { on }\end{array}$ & $\begin{array}{l}23 \\
42\end{array}$ & $\begin{array}{l}9(39) \\
9(21)\end{array}$ & $\begin{array}{l}479 \\
460\end{array}$ & $\begin{array}{l}12(2 \cdot 5)^{*} \\
12(2 \cdot 6)^{*}\end{array}$ \\
\hline $\begin{array}{l}\text { admission } \\
\text { Not responsive to penicillin treatment } \\
\text { Underlying neoplasm } \\
\text { Multiple lobes involved }\end{array}$ & $\begin{array}{l}27 \\
54 \\
19 \\
12\end{array}$ & $\begin{array}{r}10(37) \\
13(34) \\
6(32) \\
8(67)\end{array}$ & $\begin{array}{l}450 \\
448 \\
483 \\
490\end{array}$ & $\begin{array}{l}11(2 \cdot 4)^{*} \\
8(1 \cdot 8)^{*} \\
15(3 \cdot 1)^{*} \\
13(2 \cdot 7) \dagger\end{array}$ \\
\hline not rising with treatment & 9 & $4(44)$ & 493 & $17(3.4) \dagger$ \\
\hline
\end{tabular}

$* \mathrm{p}<0.001, \chi^{2}$ test.

$+\mathrm{p}<0.001$, Fisher's exact probability test. 
contribute little to the planning of initial treatment-a view which has been expressed previously. $^{78}$

The admission rate of patients with acute pneumonia was approximately twice as high at the end of the dry season as in the middle of the rainy season; this variation is similar to that observed in Nigeria. ${ }^{9}$ The reason for this is not entirely clear, though it has been suggested that drying of the upper airway due to the low humidity during the dry season may impair the local defences of the nasopharynx. ${ }^{110}$ Furthermore, food is more scarce at the end of the dry season and the resulting subtle deterioration in nutrition might further lower resistance to bacterial infection. There was also a higher incidence of tonsillitis and meningitis during the dry season in this hospital.

The observations indicate that parenteral penicillin should be the initial treatment for patients with lobar or segmental pneumonia in rural Central Africa. Since the aetiology of non-penicillin responsive pneumonia in this region is not known, it seems prudent to treat patients who do not respond to penicillin with an antibacterial combination having as wide a spectrum as local availability allows; we found the combination of penicillin with an aminoglycoside to be effective in several cases. The further addition of tetracycline was successful in a few patients, some of whom might have had mycoplasmal infection. A relatively expensive parenteral drug combination such as ampicillin and erythromycin, which has been advocated for the initial treatment of severe pneumonia in Britain, ${ }^{11}$ would be too costly for wide use in developing countries and would probably confer only a minor advantage in regions where response to penicillin is still the norm.

Not all of the patients who were unable to mount leucocyte and febrile responses to pneumonia were elderly, an observation which raises the question of whether the probably widespread suboptimal nutrition in this region is an important factor in predisposing people to pneumonia and in blunting the patients' immune response to infection. Further studies on the interplay between nutritional state, immune response, and bacterial infection in this region would help to clarify the picture and would provide a factual basis on which to plan effective primary health care schemes aimed at reducing the incidence of bacterial pneumonia.

I wish to record my thanks to Drs C Oommen, A Oommen, M Nath, and $\mathrm{H}$ Satya for their diligent care of the patients, Dr N Nath for his comments on several of the chest radiographs, Mrs JC Allen for help with filing the data, and the nursing staff at Kasama General Hospital for their devotion to duty. I was seconded to the hospital by the Overseas Development Administration.

\section{References}

' Macfarlane JT, Adegboye DS, Warrell MJ. Mycoplasma pneumoniae and the aetiology of lobar pneumonia in northern Nigeria. Thorax 1979;34:713-9.

${ }^{2}$ Greenwood BM, Hassan-King M, Macfarlane JT, et al. Pneumococcal serotypes in West Africa. Lancet $1980 ; \mathrm{i}: 360$.

${ }^{3}$ Sofowara EO, Onadeko BO. Complications and prognostic factors in pneumonia among Nigerians. Niger Med J 1973;3:144-5.

4 Tugwell DA, Greenwood BM. Pneumococcal antigen in lobar pneumonia. J Clin Pathol 1975;28:118-23.

${ }^{5}$ Douglas RM, Riley ID. Adult pneumonia in Lae-99 consecutive cases. Papua New Guinea Med J 1970;13:105-9.

- Sutton DR, Wicks ACB, Davidson L. One-day treatment for lobar pneumonia. Thorax 1970;25:241-4.

' Barrett-Connor E. The non-value of sputum culture in the diagnosis of pneumococcal pneumonia. Am Rev Respir Dis 1971;103:845-8.

${ }^{8}$ Davidson M, Tempest B, Palmer DL. Bacteriological diagnosis of acute pneumonia. Comparison of sputum, transtracheal aspirates and lung aspirates. JAMA 1976;235: 158-63.

${ }^{9}$ Warrell DA. Respiratory tract infections in the tropics. Practitioner 1975;215:740-6.

10 Brunner H, Greenberg HB, James WD, Horswood RL, Couh RB, Chanock RM. Antibody to Mycoplasma pneumoniae in nasal secretions and sputa of experimentally infected human volunteers. Infect Immun 1973; 8:612-20.

"Macfarlane JT, Finch RG, Ward MJ, Macrae AD. Hospital study of adult community-acquired pneumonia. Lancet 1982;ii:255-8. 